\title{
La Coordinación del Grado y el Plan de Acción Tutorial en la Facultad de Sociología. La necesidad de un trabajo conjunto
}

\author{
Golías Pérez, Montserrat; Santiago Gómez, Elvira
}

Facultad de Sociología, Universidade da Coruña.

\section{RESUMEN}

En los últimos tres años ha aumentado considerablemente el número de estudiantes del Grado en Sociología al mismo tiempo que ha variado la composición de la plantilla de personal docente e investigador del centro. Esta nueva coyuntura afecta tanto a las labores de gestión de la facultad, como al desarrollo del plan de estudios y la tutorización del alumnado, identificándose la necesidad de un trabajo conjunto entre el Plan de Acción Tutorial y la Coordinación del Grado en un sistema de gestión integrado. Con esta iniciativa se persigue la consolidación de una nueva cultura tutorial que evite el solapamiento de las acciones docentes y de tutorización realizadas desde el centro, al tiempo que se garantice una mayor implicación y participación activa de todos los actores que forman parte de la comunidad universitaria: equipo de dirección, personal docente e investigador, personal de administración y servicios, alumnado y servicios centrales. Es por este motivo que desde la Coordinación del Plan de Acción Tutorial y del Grado, se propone un proyecto piloto que se inicia en el presente curso 2018-19, que denominamos “Coordinación Tutorial”, que confía en una enseñanza centrada en el aprendizaje participativo y la adquisición de competencias propias y transversales, asumiendo la docencia, la gestión y la tutorización como una responsabilidad compartida.

PALABRAS CLAVE: coordinación, tutorización, aprendizaje, participación, colaboración 


\section{CITA RECOMENDADA:}

Golías Pérez, M.; Santiago Gómez, E. (2019): La coordinación del Grado y el Plan de Acción Tutorial en la Facultad de Sociología. La necesidad de un trabajo conjunto. En De la Torre Fernández, E. (ed.) (2019). Contextos universitarios transformadores: construíndo espazos de aprendizaxe. III Xornadas de Innovación Docente. Cufie. Universidade da Coruña. A Coruña (pág. 451-468).

DOI capítulo: https://doi.org/10.17979/spudc. 9788497497121.451

DOl libro: https://doi.org/10.17979/spudc.9788497497121

\section{ABSTRACT}

Since 2015 there has been a considerable increase in the number of students in the Sociology Degree at A Coruña University, at the same time the composition of the teaching and administration staff of the centre has changed. This new context affects both the management tasks of the faculty, as well as the development of the learning process and the tutorial actions. And shows the need of collaborative work between the Tutorial Action Plan and the Degree Coordination Degree in an integrated management system. The aim of this initiative is to consolidate a new tutorial culture that avoids the overlapping of teaching and tutoring actions carried, guaranteeing greater involvement and active participation of all the actors that take part of the university community: management team, teaching, administration and central services staff and students. The pilot project "Tutorial Coordination" is proposed during the present academic year from the Coordinators of the Degree and the Tutorial Action Plan, it is focused on a participated acquisition of own and transversal competences, assuming learning, management and tutorial activities as a shared responsibility.

KEY WORDS: coordination, tutoring, learning, participation, collaboration 


\section{INTRODUCCIÓN.}

En el proceso de adaptación de la Universidad española al Espacio Europeo de Educación Superior se acuerda que la función tutorial ocupe una posición preeminente para poder profundizar adecuadamente en la transmisión del conocimiento, funcionando ésta como herramienta clave en el desarrollo académico y profesional del estudiantado a través del diseño y la determinación de los objetivos personales de cada estudiante (Fernández, 2008). Esta transformación supone el abandono del modelo académico eficientista, centrado en el profesorado y caracterizado por una gobernanza jerárquica. La Universidad actual se caracteriza por una paradójica contradicción entre la tradición y la innovación (Hípola y Río, 2005), en la que los modelos de enseñanza centrados en el docente y con una concepción del conocimiento cerrada, han dado paso a una nueva realidad en la que existe un compromiso creciente por la recuperación del carácter humanístico de la enseñanza superior, su papel social y su compromiso en la construcción de un mundo justo. Para ello es necesario formar al alumnado desde una perspectiva crítica y centrada en su tutorización, reivindicando un modelo de enseñanza-aprendizaje más abierto a la diversidad (Torrego y Ruiz, 2011).

El Plan de Acción Tutorial y la Coordinación de la titulación deben capacitar al alumnado a alcanzar sus objetivos académicos y profesionales a través de la orientación (Fernández y Medialdea, 2013). Jesse B. Davis, pionero en la integración de la orientación del alumnado en el programa educativo, consideraba que la meta del sistema universitario es logar que el alumnado adquiera una mejor comprensión y dominio de su carácter haciéndole consciente del contexto social que le rodea (González, 2006).

La orientación a través de la tutorización debe contribuir al desarrollo de competencias y habilidades relacionadas con la resolución de conflictos, el autoconocimiento, la responsabilidad, la empatía, el liderazgo, el desarrollo de la capacidad de reflexión, etc. (González y Pedro, 2013). La tutoría, burocrática, académica 0 de asesoramiento es una función constante en toda actividad docente (Lázaro, 2002). Entre los roles y funciones que la tutorización desempeña, lo más importante, y a la vez lo más complicado, es la adaptación a 
las características del alumnado, por ello requiere de un arduo trabajo por parte del personal docente y de administración del centro, orientado a conocer las necesidades de sus estudiantes para poder generar un entorno de aprendizaje que fomente el desarrollo comunitario (Sanz et al, 2009).

Para aproximarnos al contexto que nos ocupa, desde el año 2015, la Facultad de Sociología de la Universidade da Coruña (UDC) ha experimentado importantes transformaciones. Por un lado, la composición del Personal Docente e Investigador ha variado considerablemente con la incorporación de nuevo profesorado contratado y con la llegada de investigadores bajo programas posdoctorales. Además, también ha habido modificaciones entre el Personal de Administración y Servicio a lo que se le suma un importante incremento en el volumen de estudiantes matriculados (véase Gráfico 1), lo que supone un reto que solo puede asumirse mediante una estrecha colaboración entre todos los agentes que forman parte del centro y a través de una estrategia de trabajo conjunto.

\section{Gráfico 1. Evolución del número de estudiantes en el Grado de Sociología}

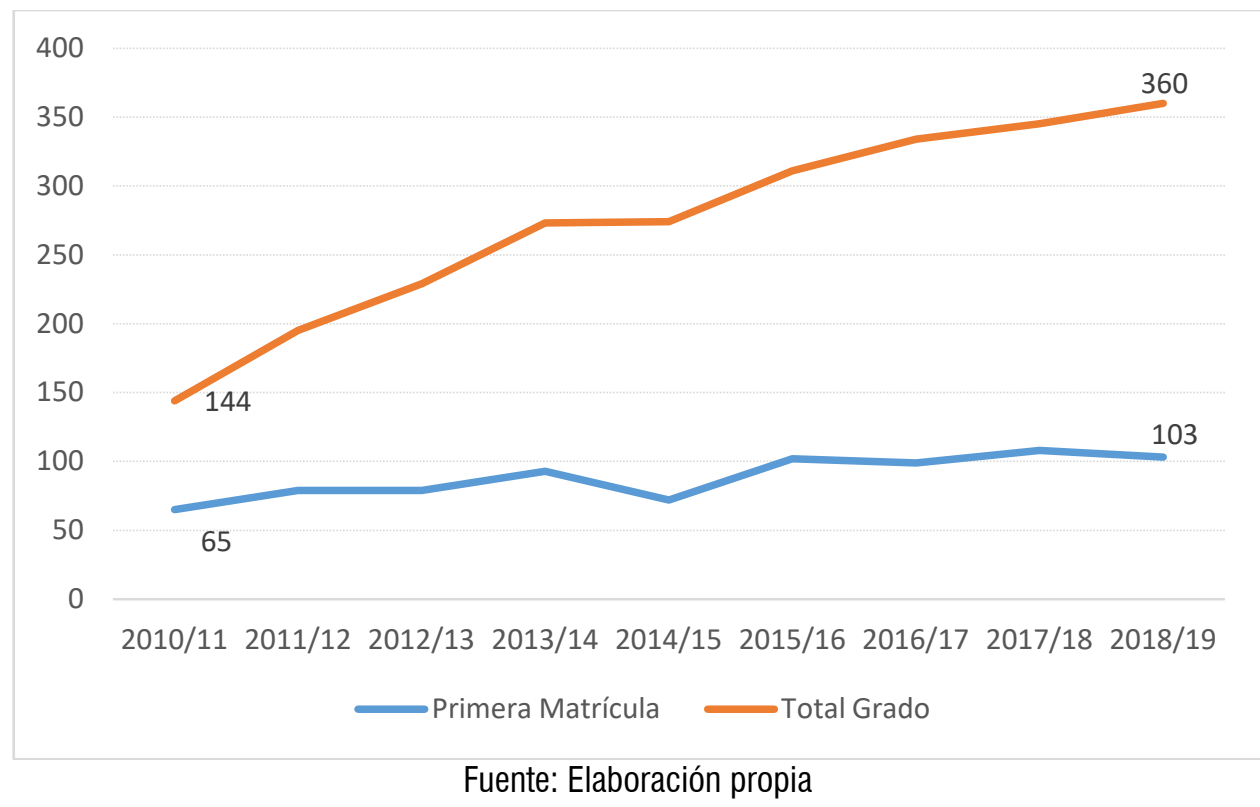




\section{DIAGNÓSTICO: LA DEBILIDAD DE LOS CANALES DE COMUNICACIÓN}

Si se tienen en cuenta los indicadores de calidad derivados de la satisfacción del alumnado, uno de los principales puntos débiles de la titulación se refiere a la tasa de abandono. Reforzar las labores de tutorización, especialmente durante el primer curso del grado, se convierte en una estrategia prioritaria para revertir esta situación. Desde el centro se han realizado reajustes en la organización de la docencia y se han introducido nuevas figuras para complementar la tutorización entre las que destaca, por su éxito, la figura de "coordinadores de curso". En atención a estas medidas se han incorporado jornadas informativas, organizadas desde la coordinación del grado y del prácticum, así como tutorías colectivas con el alumnado focalizadas en las temáticas de interés específico para cada curso. La evolución de los datos muestra un descenso significativo de la tasa de abandono en el último año académico 2016/17, con un 9,85\% (7 abandonos en el total de 71 estudiantes). A pesar de este éxito y con la intención de afianzar los buenos resultados, es necesario prestar atención al alumnado también en el último tramo de la carrera, organizando charlas específicas sobre el programa de prácticas, el trabajo de fin de grado, o las salidas profesionales, a fin de conocer cuáles son los inconvenientes que dificultan la continuación de los estudios y orientarles en la etapa que se inicia con su entrada en el mercado laboral. Estas medidas se espera que repercutan positivamente en la tasa de graduación, que en el curso 2016/17 se encontraba en el 33,7\%', una cifra que debe aumentar si bien es cierto que se detecta una mejoría considerable respecto a cursos anteriores, como constata la subida de cuatro puntos porcentuales desde el curso 2012/2013.

Continuando la aproximación a la realidad del centro, cabe mencionar que el grado de satisfacción del alumnado de primer curso con las acciones de orientación ha experimentado un ligero incremento, aunque se mantiene por debajo de las expectativas, con una puntuación de 3,76 sobre 7, frente al 3,46 sobre 7 del curso 2015-16. El Gráfico 2 recoge la evolución de la satisfacción del alumnado de primer curso, que como se observa ha sufrido ligeros 
descensos seguidos de leves recuperaciones y oscila entre su mínimo en 3,19 en el curso 2014-15 y su máximo en el curso 2016-17.

Gráfico 2. Evolución del grado de satisfacción de orientación de los estudiantes de $1^{0}$ curso

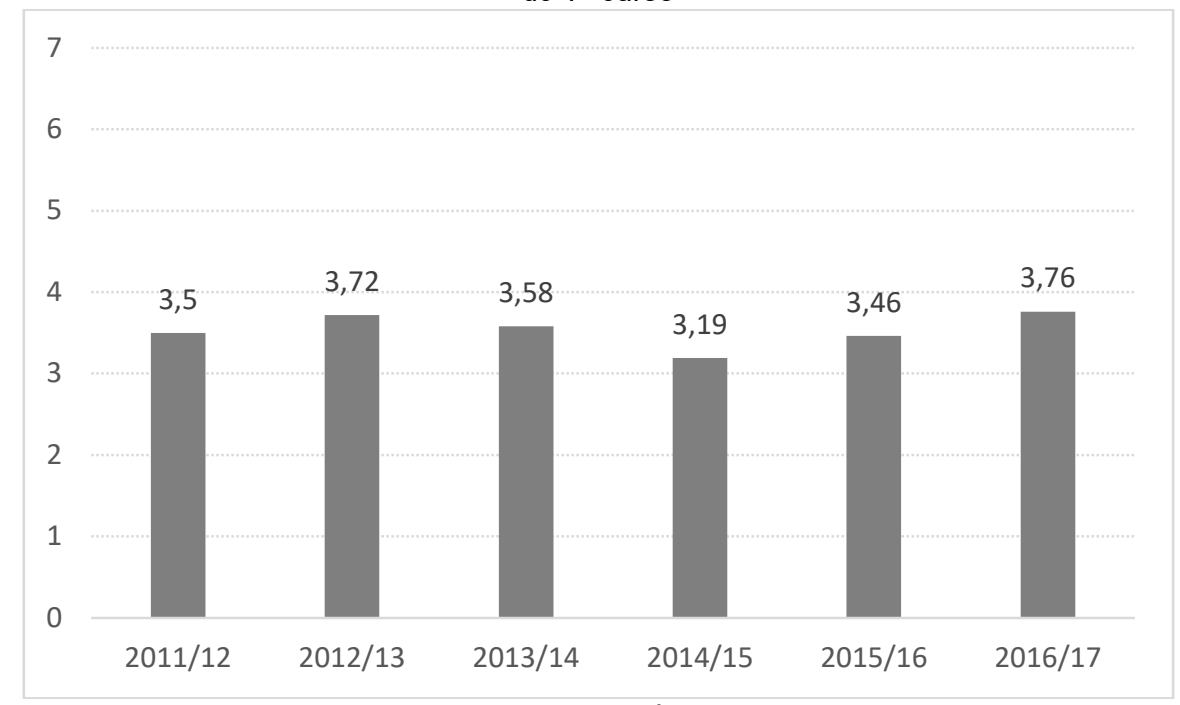

Fuente: Elaboración propia

En la satisfacción expresada por el alumnado de $3^{0}$ la situación es diferente pasando de 2,71 en el curso 2015-16 a 3,93 en el año académico 2016-17. Esta mejora en la valoración coincide con la incorporación del Plan de Coordinación Horizontal, que cuenta con las ya mencionadas figuras de "coordinador/a de curso", además de los "delegados de curso", implantadas en el año 2014/2015, pero desvinculadas del Plan de Acción Tutorial. El impacto positivo de estos agentes de mediación se asocia a la interiorización del alumnado de los nuevos canales de comunicación con el centro, útiles en la resolución de conflictos, la obtención de información y la atención en aspectos relacionados con la docencia. Una de las actividades que se han puesto en marcha a través de la coordinación horizontal, han sido las "tutorías colectivas" para atender los requerimientos del alumnado que posteriormente eran puestos en común en las reuniones de coordinación cuatrimestrales, con la intención de dar respuesta. 
No obstante, y habida cuenta del éxito de la implantación de estas nuevas figuras y formas de organización, se persigue alcanzar una mejora significativa de los indicadores manteniendo el sistema de coordinación horizontal, conectándolo con el resto de órganos y agentes coordinadores del centro y tratando de adaptarlo a la nueva realidad del centro.

Tras la revisión de estos indicadores, la facultad debe adecuarse a la nueva realidad para poder seguir respondiendo a las necesidades y demandas del estudiantado de Sociología, ante este nuevo panorama.

Al margen de los resultados numéricos, las siguientes observaciones nos invitan a replantearnos el Plan de Acción Tutorial, tal y como se aplicaba hasta la fecha (curso 2017/18), que contaba con una serie de herramientas o figuras que se recogen a continuación.

1. Coordinador del PAT

2. Tutorización de Profesores

3. Tutorización de alumnos/as

4. Coordinadores de Curso

5. Tutorías colectivas

6. Jornada de Acogida

7. Representantes de alumnos/as

8. Jornadas informativas a alumnado potencial

9. Tutorías académicas

10. Materiales y herramientas protocolizadas

La observación y análisis de su eficacia evidencia los siguientes inconvenientes:

- Los/as "coordinadores/as de curso", así como las "tutorías colectivas", aunque se encontraban dentro de la Acción Tutorial, dependían de la Coordinación del Grado, sin implicación directa por parte de la Coordinación del PAT que era responsable de la asignación de tutores-profesores. 
- Escasa respuesta del alumnado a las convocatorias realizadas por el/la tutor/a asignado desde el PAT. Se ha detectado que el alumnado no acudía al profesor-tutor para el asesoramiento, orientación o resolución de posibles conflictos. Esta figura era sustituida por la del Coordinador/a de Curso (perteneciente a la Coordinación Horizontal), los/as delegados/as, los representantes de estudiantes o lo que venimos en denominar "tutores informales", es decir aquellos profesores con los que se encontraban más cómodos.

- El papel de los coordinadores de curso, que funciona exitosamente, se solapaba en múltiples ocasiones con las tareas que debería realizar el Tutor/a-Profesor/a.

- La tutorización por parte de la figura del "alumno/a-mentor", se había iniciado como prueba piloto en el año académico 2015-16, pero no se ha vuelto a activar.

- Las tutorías colectivas han sido la herramienta fundamental para la recogida de información sobre la situación del alumnado de Sociología. No obstante, el nivel de participación del estudiantado es bajo, y disminuye a medida que avanzan los cursos académicos.

- La orientación recibida con relación al grado durante la Jornada de Acogida es considerada insuficiente por el alumnado de nuevo ingreso.

- En cuanto a las jornadas informativas al alumnado potencial, la participación en programas como, Visita o teu Campus! Promovidos por el Servicio de Asesoramiento y Promoción del Estudiante (SAPE) de la Universidad de A Coruña, no permiten informar de manera aceptable sobre el grado. Por lo que habría que pensar en otras actividades para la promoción del título.

- Escasa o nula participación de los pocos representantes de estudiantes que hay en el centro (en la actualidad la facultad solo cuenta con 2 representantes de alumnos/as)

- Materiales y herramientas protocolizadas. Son de gran ayuda para la aplicación del PAT, pero la escasa respuesta del alumnado nos invita a reflexionar sobre ellas. Entre los materiales contábamos con un cuestionario de acogida, y encuestas que evalúan 
la satisfacción de las personas matriculadas en el centro, en las que se deberían incorporar nuevos indicadores. Por otro lado, la "carpeta de estudiante" no ha resultado una guía utilizada por el alumnado.

Tras esta revisión y ante la nueva situación del centro, se plantea para el éxito de aplicación del Plan de Acción Tutorial, vincularlo con el Sistema de Coordinación Horizontal, dependiente de la Coordinación del Grado, confiriendo mayor importancia la figura del Coordinador de Curso. Esta será la persona de referencia, a la que deberá acudir el alumnado quién recibirá las consultas y las derivará según sea el caso al profesor/a-tutor/a, a la Comisión de Estudiantes (alumnos/as-mentores/as), o al servicio del centro que resulte más apropiado.

\section{EL SISTEMA DE COORDINACIÓN TUTORIAL: UNA PROPUESTA DE TRABAJO CONJUNTO}

Revisar cuales han sido las fortalezas y debilidades en el funcionamiento de una facultad como la de Sociología de A Coruña, nos ha devuelto a la teoría, que transmitimos en las aulas de nuestro grado, pero que no aplicamos en la articulación de nuestra organización. Nos centramos en un modelo tradicional, centrado en la productividad, obviando una de las aportaciones fundamentales de la sociología al estudio de las organizaciones "el interés por los procesos sociales que ocurren en su interior para alcanzar una comprensión más completa de la vida organizativa" (Lucas Marín, 2013:18) y en esta visión amplia es en la que se basa nuestro proyecto piloto. Se trata de un sistema de coordinación integral, que cuenta con una estructura en la que se integran de forma activa: la dirección do centro, la coordinación del grado, la coordinación del PAT, el profesorado, el alumnado y el personal de administración y servicios.

La adecuada organización del centro, la satisfacción laboral del personal (PDI y PAS), así como el éxito de la trayectoria del alumnado son realidades dependientes que requieren de una estrategia de compromiso compartido, de la que parte este proyecto, centrada en establecer canales de comunicación. 


\subsection{FIGURAS IMPLICADAS EN ESTE NUEVO MODELO DE COORDINACIÓN}

El equipo decanal, apoyará el desarrollo de este Plan, proporcionando no solo las infraestructuras y recursos necesarios, sino también dando respuesta a las consultas y peticiones transmitidas por los responsables de la coordinación tutorial (coordinación del grado y del PAT).

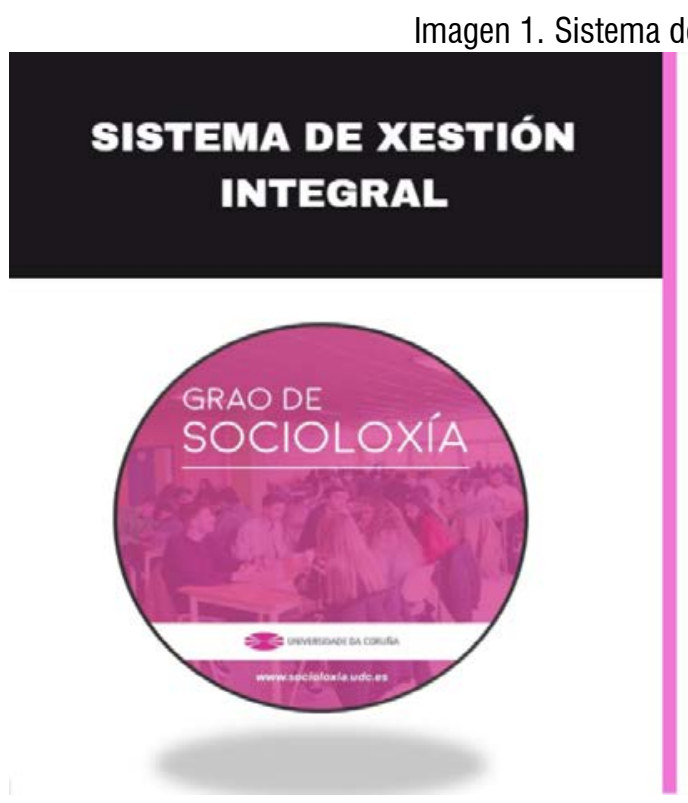

\author{
ORGANOS DE GOBERNO DA \\ FACULTADE DE SOCIOLOXIA
}

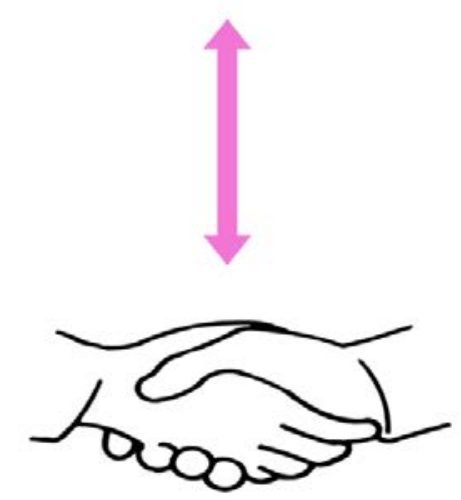

COORDINACIÓN DE GRAO - PAT

Para su funcionamiento, la Coordinación Tutorial, contará con la colaboración de todas las personas con funciones de coordinación en el centro (coordinación de movilidad internacional, prácticum, TFGs y coordinadores de curso) y cuando sea preciso del personal de Administración (PAS), y de otros servicios de la Universidad (OIXii, ADliii y UDC Saludable $\left.^{\text {iv }}, \ldots\right)$. Pero tratará de implicar también a otros órganos de la facultad que hasta la fecha funcionaban de manera independiente (Departamento), o no existían (comisión de estudiantes).

Es preciso establecer un sistema en el que fluya la información y con unas funciones claras para el éxito de esta propuesta. Para ello se cuenta con profesores/as y alumnos/as que 
llevarán a cabo estas acciones de mediación entre el estudiantado y la coordinación tutorial, que posteriormente servirá de correa transmisora con el servicio correspondiente y con la dirección del centro. A continuación, se detallan los perfiles y roles de los principales receptores de lo que sucede en la facultad:

Los/as profesores/as-coordinadores. Tendrán un papel relevante en la implementación de esta propuesta:

- Atención al estudiantado, recepción de las demandas, que serán transmitidas a la coordinación tutorial.

- Gestionar las tutorías colectivas y las reuniones con agentes implicados en la tutorización del nivel correspondiente: profesores/as-tutores/as, alumnos/asmentores/as y delegados/as de grupo.

- Hacer un seguimiento o evaluación cuantitativa y cualitativa de la marcha del curso del que son interlocutores/as.

Los/as profesores-tutores/as, sus funciones están recogidas en el Plan de Acción Tutorial (PAT) del CUFIE ${ }^{\vee}$. Su papel consiste en guiar e informar al estudiante en aspectos más individuales relativos a la trayectoria académica, profesional y personal, ya que los generales 0 comunes a todo el estudiantado serán abordadores desde la coordinación del curso. A la luz del análisis del Plan de Acción Tutorial y ante el fracaso en el sistema de asignación automática de tutores/as, se incluye la siguiente novedad con respecto a años anteriores. Será el alumno/a quien demande este tipo de tutoría personalizada, dirigiendo sus solicitudes al coordinador/a de curso en el que estén matriculados/as con mayor número de créditos. La lista de tutores/as estará a disposición del alumnado, quién podrá manifestar su interés por un/a profesor/a concreto o en caso contrario desde la coordinación del PAT se le asignará el que mejor encaje con el tipo de demanda y el perfil del estudiante.

En cuanto a los/as tutores específicos, los/as estudiantes contarán con un/a tutor/a de movilidad, destinado/a al asesoramiento del alumnado de intercambio. Pero en el caso del 
alumnado que requiera "atención a la diversidad" (ADI), con la intención de normalizar su situación y de no ser identificados por el resto de compañeros/as, no habrá un tutor específico de ADI. Los alumnos/as con esta condición serán guiados por tutores/as-profesores/as, que dispondrán del apoyo y asesoramiento necesario por parte de la coordinación del PAT y del servicio que pone a disposición el CUFIE de nuestra universidad. En el caso de los deportistas de "alto nivel" el procedimiento será similar. De esta forma se espera implicar a todo el profesorado que participa en las labores de tutorización en las demandas del alumnado, sean del tipo que sean.

Imagen 2. Dar respuesta a las demandas y necesidades del alumnado/a

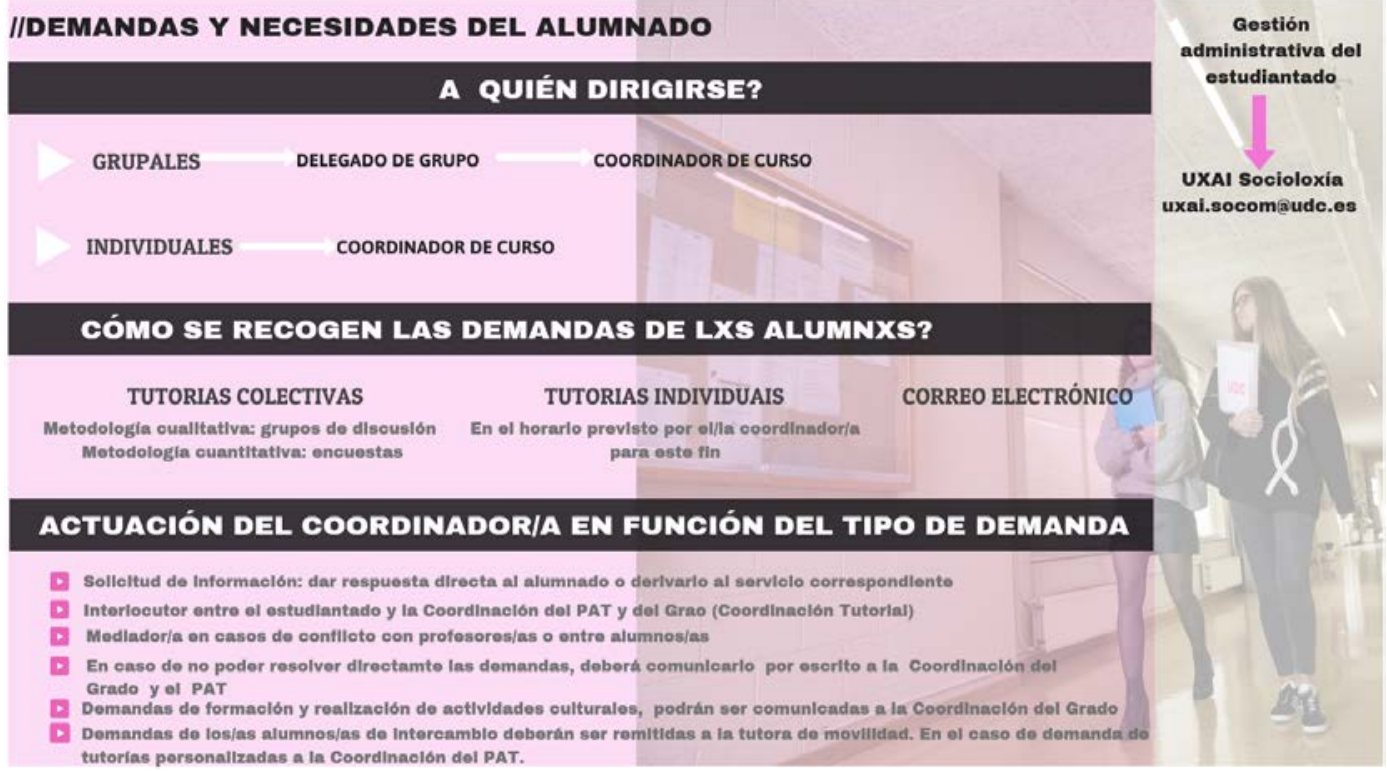

Se pretende retomar la figura del alumnado-mentor, que también recoge el Plan de Acción Tutorial del CUFIE. Dada la escasa representación estudiantil en el centro se intenta impulsar a partir de esta figura y su implicación en la vida del centro un tejido participativo. Será desde los/as alumnos/as de los dos últimos niveles del grado sobre los que recaerá esta responsabilidad. La labor de la Coordinación Tutorial será informativa y de motivación a partir 
de la cual se promoverá la autogestión por parte de los estudiantes, sin intermediación de la coordinación de curso.

Se fomentará la constitución de una Comisión de Estudiantes, que elegirá a dos representantes, que formarán parte del trabajo de evaluación de la eficacia de esta propuesta, además de las actividades que consideren de interés para el alumnado del centro. Con esta iniciativa la intención es establecer nuevas vías de comunicación con el estudiantado ante el vacío de representación de este colectivo en la actualidad. Los alumnos/as-mentores/as, miembros de esta Comisión, servirán de referentes para aquellos/as de nuevo ingreso y podrán orientarles en aspectos de la vida académica, también formarán parte de las Jornadas de Bienvenida del centro para los recién matriculados/as y participarán en las actividades de promoción del título. Desde la Coordinación Tutorial y la dirección del propio centro se les animará a ser proactivos gestionando de manera autónoma sus reuniones y proponiendo iniciativas.

Se importa a este nuevo sistema y se vincula con el PAT a los/as delegados/as, fruto de la Coordinación Horizontal puesta en marcha en años anteriores. Habrá un delegado por grupo interactivo y no por curso (ya que la casuística puede variar debido a que tienen horarios y en ocasiones profesores diferentes). A pesar de reforzar la representación estudiantil por medio de la comisión de estudiantes y de reactivar el mentoring, no es posible prescindir de los delegados/as que actúan como interlocutores entre sus compañeros y la coordinación 0 incluso la propia comisión de estudiantes. Entre sus obligaciones se encuentran: participar activamente en las tutorías colectivas y reuniones de coordinación; dinamizar y difundir las actividades formativas e informativas organizadas desde la coordinación.

\subsection{SISTEMA DE EVALUACIÓN: LA IMPORTANCIA DEL PROCESO}

Además del tradicional sistema de evaluación mediante encuestas como son las de Acogida, el PAT o las del Sistema Interno de Garantía de Calidad (SIGC) de la universidad, las tutorías colectivas se convierten en la clave, no solo a la hora de establecer un feedback con el 
alumnado, sino también como un sistema de evaluación cualitativo de su grado de satisfacción. Retomando a Lázaro, podemos afirmar que en las actividades de acción tutorial en el entorno educativo, como las que se recogen en este sistema de coordinación, "el producto formativo es el propio proceso, y no el mero resultado" (Lázaro, 2008:112).

El análisis de los datos recabados a través de la metodologías cualitativa y cuantitativa debe realizarse también de forma participativa. Para ello se propone la creación del "Grupo de Trabajo de Coordinación Tutorial” (GTCT), del que formarán parte la Coordinación del Grado y del PAT, además de los coordinadores de curso y dos representantes elegidos por los alumnos-mentores (pertenecientes a la comisión de estudiantes).

Imagen 3. Funciones del Grupo de Trabajo de Coordinación Tutorial.

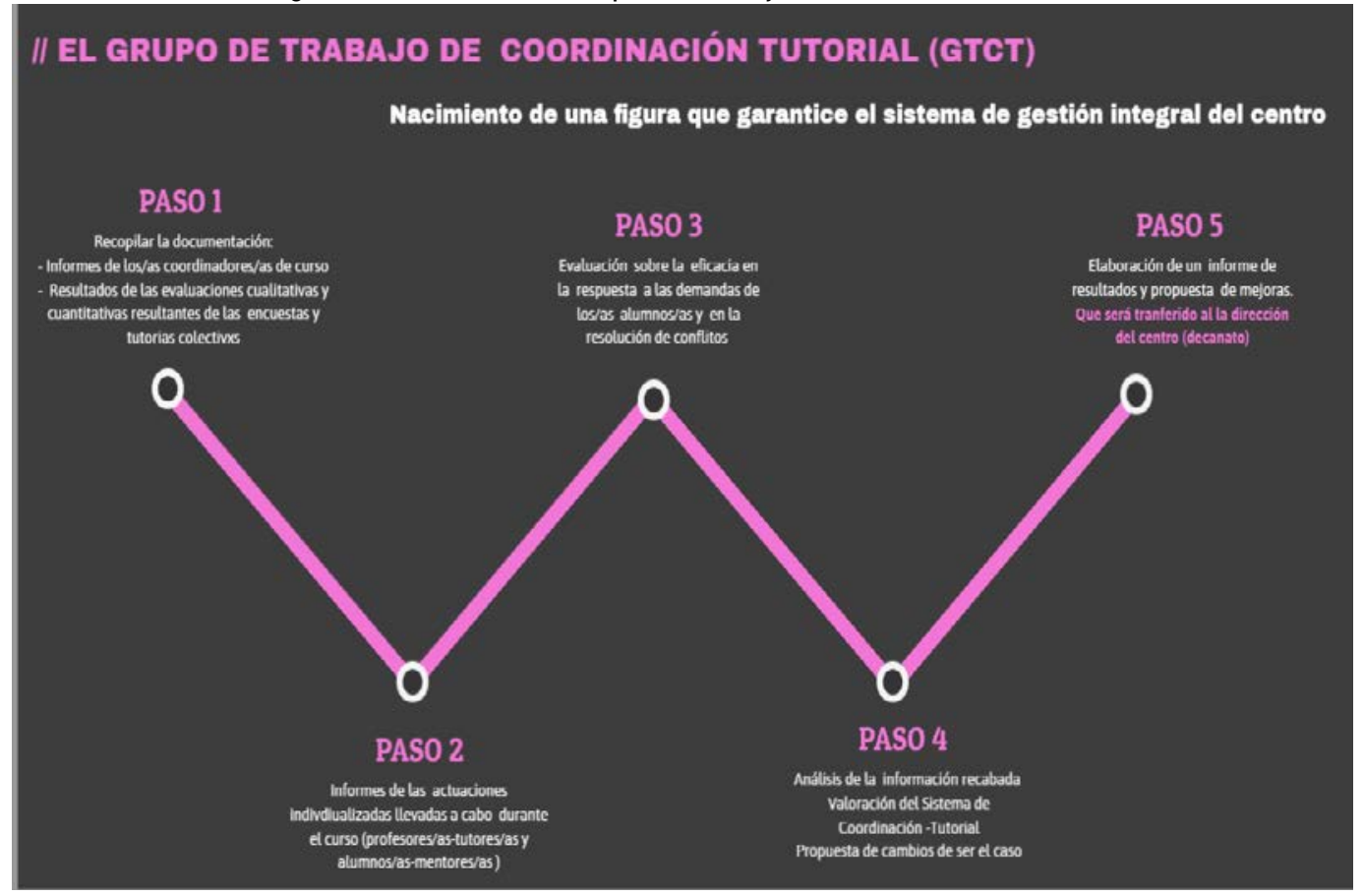

Al margen de las figuras que acabamos de señalar y de las herramientas de evaluación, el sistema de coordinación tutorial cuenta con una serie de actividades para su aplicación, a las que se añaden también jornadas y talleres formativos generales o específicos enfocados a los 
diferentes cursos del grado. Además, se ampliarán las Jornadas de Acogida, en cuanto a su duración y a los contenidos que ofrecían tradicionalmente, en lo que se denominará "Semana 1" (semana de acogida).

Imagen 4. Actividades llevadas a cabo en el marco del Sistema de Coordinación Tutorial, curso 2018-19.

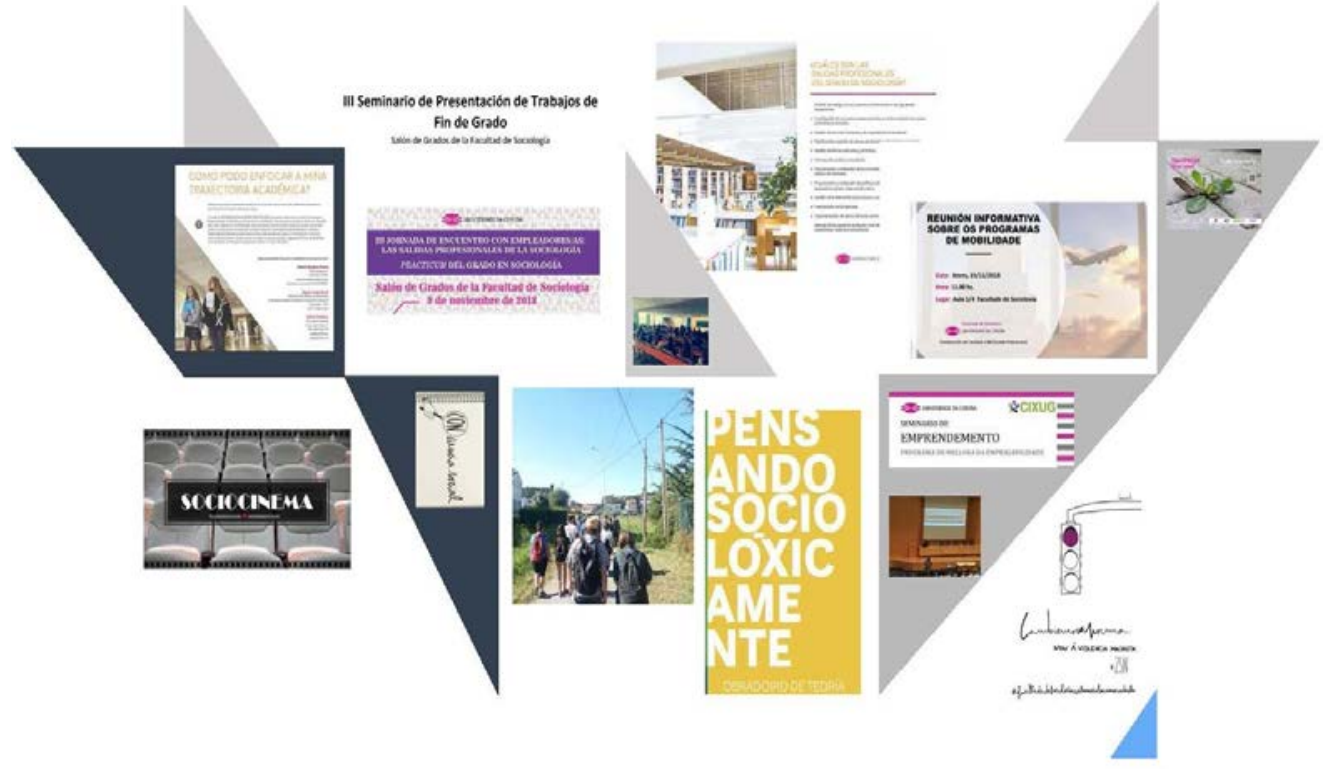

\section{PRIMEROS RESULTADOS Y CONCLUSIONES}

Reorganizar las tareas de Coordinación del Grado y el Plan de Acción Tutorial requiere de la implicación del PDI y PAS. En la actualidad, la precariedad e inestabilidad en la que se encuentra el profesorado, obliga a tener únicamente en cuenta un sistema de calidad cuando hablamos de la academia, el de la productividad científica. Imbuidos en los criterios de evaluación de nuestra actividad investigadora, ignoramos otros aspectos clave que repercuten en la calidad universitaria, como son la gestión y la docencia, con esta propuesta se promueve la conexión entre estos tres polos de la vida académica. En lo que se refiere al Personal Administrativo y de Servicios del centro, las personas que trabajan en la biblioteca sí han interiorizado esta participación en los procesos de aprendizaje fuera de las aulas, y entendido 
la vida universitaria como un proyecto de comunidad del que todos formamos parte, esta visión debería extrapolarse al resto del personal de administración de la facultad, que está en contacto directo con el alumnado como receptor de sus tramitaciones y solicitudes de carácter burocrático.

Entre el alumnado, la tendencia semeja similar, atender a los resultados académicos les aleja de la participación, de la vida en comunidad y de una relación con el aprendizaje que va más allá de lo estrictamente curricular. Será gracias a las labores de orientación como se alcanzarán los objetivos que persigue este nuevo sistema de coordinación: reducción de la tasa de abandono, el aumento de la satisfacción y la implicación de los estudiantes en las actividades propuestas. Este acercamiento solo se alcanzará si se reducen las barreras comunicativas entre alumnado y profesorado, bien abriendo espacios físicos para la interacción, como las tutorías colectivas, así como espacios virtuales a través de las redes sociales, adaptándonos a sus formas de comunicación.

Aunque la propuesta que aquí se recoge es de reciente implantación, este proyecto piloto ya cuenta con resultados inmediatos que se traducen en:

- Un incremento del alumnado en la participación en las tutorías colectivas y en la primera reunión de coordinación.

- La organización de una Comisión de Estudiantes, a partir de los/as mentores/as que ya se está autogestionando y comunicando con las personas matriculadas en primero y segundo curso del grado.

- El afloramiento de realidades que estaban veladas: deportistas de alto nivel que requieren de una adaptación de sus planes formativos, la necesidad de atender a las cuestiones relacionadas con la de identidad de género, a través de la sustitución del nombre registral por el nombre sentido en los canales de comunicación oficiales; 0 la atención a la diversidad funcional, de manera que nuestros/as alumnos/as cuenten con un ambiente de aprendizaje adecuado disponiendo de un contacto social favorecedor de la inclusión (Sallán et al, 2017) 
Los primeros pasos de este proyecto no hubiesen sido posibles sin el apoyo de la dirección del centro y sin el asesoramiento constante del personal de los servicios del CUFIE, además de otros agentes implicados en esta visión de universidad, y que nos ayudarán a conseguir que la facultad de sociología sea mucho más que un grado.

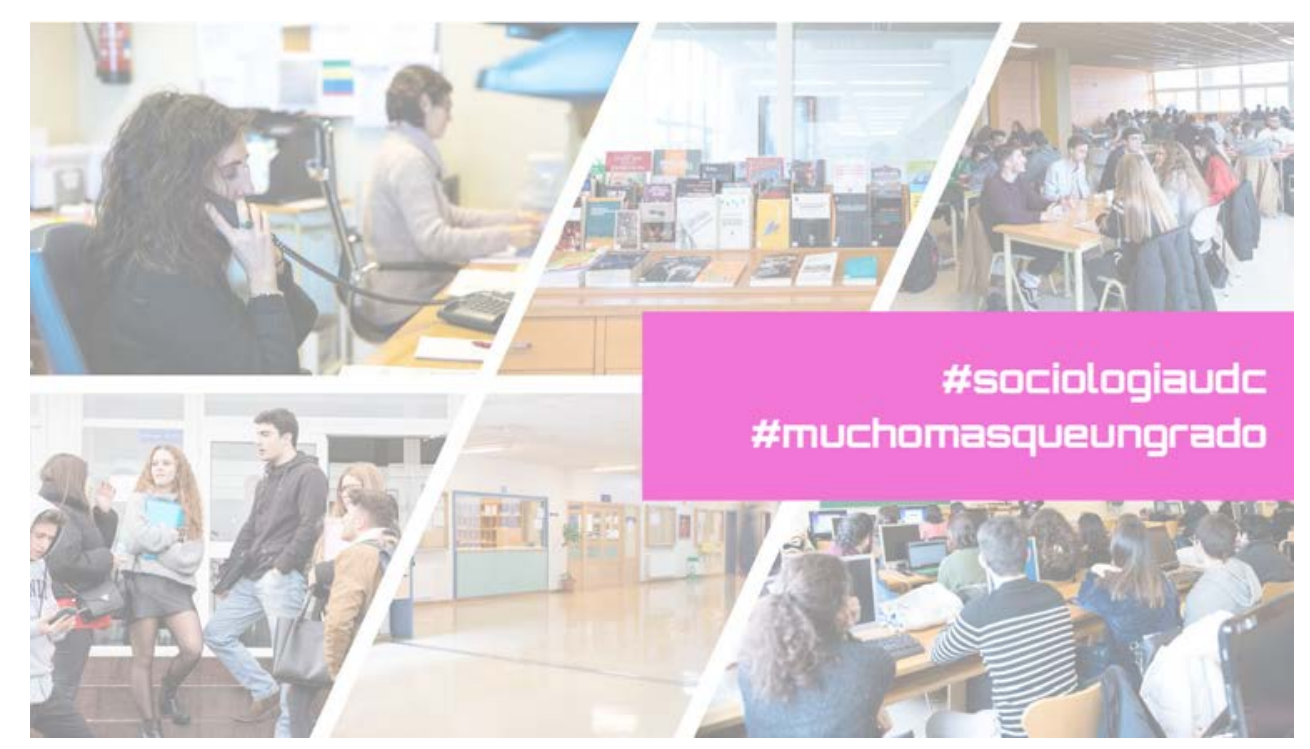

\section{REFERENCIAS}

Fernández, G. D., Bonilla, F. J. Á., \& Medialdea, A. M. L. (2013). Acción tutorial y orientación en el periodo de transición de la educación secundaria a la universidad: La orientación al alumnado de nuevo ingreso. REDU: Revista de Docencia Universitaria, 11(2), 221.

Fernández, L. M. S. (2008). Plan de acción tutorial en los centros docentes universitarios: el rol del profesor tutor. Revista interuniversitaria de formación del profesorado, (61), 89-108. González, M. Á. (2006). La acción tutorial: su concepción y su práctica. Ministerio de Educación.

González, R. C., \& Pedro, A. P. (2013). Programa Orienta: Plan de acción tutorial universitaria para estudiantes de primer curso. Contextos Educativos. Revista de Educación, (11), 161-180. 
Hípola, P. S., \& del Río, A. Z. (2005). Las universidades españolas ante el proceso de convergencia europeo: análisis de las medidas institucionales y acciones de aplicación y coordinación. Revista de educación, (337), 169-187.

Lázaro, A. (2002). La acción tutorial de la función docente universitaria. VíCTOR ÁLVAREZ ROJO \& ÁNGEL LÁZARO (coords.), Calidad de las universidades y orientación universitaria. Málaga: Ediciones Aljibe, 249-282.

Lázaro, A. (2008). Diferencias cualitativas entre experiencias tutoriales para opciones de aprendizaje universitario. Revista Interuniversitaria de Formación del Profesorado 61, 109138.

Lucas Marín, A. (2013). Sociología de las Organizaciones. Influencia de las tecnologías de la información y la comunicación. Madrid: Fragua.

Sallán, J. G., Moreno, J. L. M., Galán-Mañas, A., Rodríguez, M. F., \& Gavaldà, J. M. S. (2017). El plan de acción tutorial para estudiantes universitarios con discapacidad. Revista de Educación Inclusiva, 6(3).

Sanz Oro, R., Fernández, L. M. S., Reyes, M. L., \& Acosta, J. M. (2009). Tutoría y atención personal al estudiante en la universidad. Ed. síntesis.

Torrego Egido, L., \& Ruiz Esteban, C. (2011). La coordinación docente en la implantación de los títulos de Grado. Revista electrónica interuniversitaria de formación del profesorado, 14(4).

\footnotetext{
' Se ha observado una inconsistencia entre estos resultados y el resto de los indicadores. Por lo que se revisarán los datos originales proporcionados por los responsables de calidad en la UDC.

ii OIX, Oficina de Igualdad de Género de la Universidad de A Coruña.

iii ADI, Unidad Universitaria de Atención a la Universidad de la Universidad de A Coruña

iv UDC Saludable, Servicio de la Universidad de A Coruña, que lleva a cabo actividades que favorezcan la vida saludable en la comunidad universitaria.

v CUFIE, Centro Universitario de Formación e Innovación Educativa de la Universidad de A Coruña.
} 